\title{
Condiciones de trabajo de las enfermeras y las formas de contratación en Medellín, Colombia
}

\author{
Working conditions of nurses and the forms of contracting in Medellín, Colombia \\ Marleny Zapata-Herrera', Nora Eugenia Zapata-Gómez² \\ 1 Magíster en Salud Colectiva, Docente Facultad de Enfermería, Universidad de Antioquia. Medellín, Colombia. \\ 2 Magíster en Enfermería, Docente Facultad de Enfermería, Universidad de Antioquia. Medellín, Colombia.
} e-mail:marlenyzh@hotmail.com e-mail: norazago@udea.edu.co

Fecha de recepción: Diciembre 09 - 2014

Fecha de aceptación: Septiembre 18 - 2015

Zapata-Herrera M, Zapata-Gómez NE. Condiciones de trabajo de las enfermeras y las formas de contratación en Medellín, Colombia. Univ. Salud. 2015;17(2): 212 - 223. DOI: http://dx.doi.org/10.22267/rus.151702.6

\begin{abstract}
Objetivo: Describir las condiciones de trabajo y de organización del profesional de enfermería en diferentes formas de contratación laboral. Materiales y métodos: Estudio descriptivo, transversal, con selección de 552 enfermeras que laboraban en instituciones de $2^{\circ}, 3^{\circ}$ y $4^{\circ}$ nivel de atención en salud en una ciudad colombiana; con un tamaño muestral por conglomerados representados por 31 IPS. Se usó el cuestionario de Condiciones de Trabajo (qCT) validado para Latinoamérica. Resultados: Predominaron las enfermeras con edades entre 25 y 34 años (55\%), solteras (52\%), en instituciones privadas laboraba el $63,5 \%$, en modalidad de contrato a término indefinido $51 \%$. El promedio de valoración de las condiciones de trabajo y de organización fue positiva en todas las escalas qCT acorde con la media y desviación estándar. El tipo de contratación tiene peso en la determinación de las condiciones de trabajo, pero no es el único indicador. Conclusión: Las mejores condiciones de trabajo y de organización como mayor antigüedad laboral, mayor salario, cubrimiento total de la seguridad social y prestaciones sociales, se dan en las enfermeras que laboran en contrato a término indefinido y en empresas de carácter privado.
\end{abstract}

Resumen

Palabras clave: Condiciones de trabajo, organizaciones, enfermeras, contratos. (Fuente: DeCS, Bireme).

\begin{abstract}
Objective: To describe the conditions of work and organization of professional nursing in different forms of contracting. Materials and methods: A descriptive, cross-sectional study was made by selecting 552 nurses who were working in institutions of $2^{\circ}, 3^{\circ}$ and $4^{\circ}$ health care level in a Colombian city; with a sample size for conglomerate represented by 31 IPS. The questionnaire on working conditions (qCT) validated for Latin America was used. Results: Nurses aged between 25 and 34 years (55\%) prevailed, those who were single $52 \%$, in private institutions $63.5 \%$ and with an indefinite-term contract $51 \%$. The average assessment of the conditions of work and organization was positive in all scales qCT according to the mean and standard deviation. The type of hiring is important in the determination of working conditions, but it is not the only indicator. Conclusion: The best conditions of work and organization such as greater seniority, higher salary, total coverage of the social security and social benefits, are given to nurses who work in indefinite-term contract and private companies.
\end{abstract}

Keywords: orking conditions, organizations, nurses, contracts. (Source: DeCS, Bireme). 


\section{Introducción}

Las tendencias modernas de la globalización y los asuntos políticos y económicos del mercado laboral se caracterizan por cambios en los procesos productivos, en las relaciones organizacionales del trabajo y el surgimiento de nuevos marcos jurídicos que flexibilizan el salario, la jornada, la contratación y las condiciones de trabajo (CT) como una estrategia económica que privilegia la rentabilidad del sistema, la individualización y la incertidumbre del individuo por encima del bien común y solidario. ${ }_{1}$

Según Blanch, las CT y organización laboral son "el conjunto de circunstancias ecológicas, sociales, económicas, jurídicas, tecnológicas, etc. en el marco de las cuales se desarrolla la actividad laboral", que inciden sobre la dinámica organizacional, las relaciones laborales, la calidad de vida en el trabajo, la motivación, la satisfacción y la productividad de las personas y las organizaciones. Para Davis y Newstrom,,$_{3}$ la organización del trabajo, debe reflejar la relación armoniosa entre el administrador y el trabajador para el logro de objetivos comunes.

En Latinoamérica y el Caribe, la figura de trabajo asociativo y colectivo se ha distorsionado y las empresas pasaron a ser intermediarias de mano de obra o empresas tercerizadas, que vulneran el trabajo decente. $_{4-6}$ Las CT en enfermería se caracterizan por realidades desfavorables de contratación como: bajos salarios, incremento de la carga de trabajo, entornos físicos negativos, desmotivación, insatisfacción y abandono de los cargos. $_{7-10}$

El estudio de Baraldi y $\mathrm{Car}_{1_{11}}$ muestra que la contratación flexible en enfermería afecta sus derechos laborales, privilegia el trabajo individual calificado y el rol polivalente, donde importa más el control y el indicador, que la calidad de los procesos y los servicios.

Contrario a lo que se observa en Latinoamérica donde la mayoría de los estudios, sobre las CT de las enfermeras, resaltan aspectos desfavorables; investigaciones en Norteamérica dan cuenta de unas CT favorables y destacan además, la seguridad de la enfermera y del paciente, una mayor calidad de la atención de enfermería, así como la satisfacción y retención laboral de estos profesionales en hospitales magnéticos que reportan mejores condiciones de trabajo y menor movilidad y abandono de los cargos. $_{12-13}$ Para el caso de las enfermeras, estos entornos armoniosos de trabajo son un punto importante de intervención en la crisis del recurso humano en salud. ${ }_{14}$

En Colombia las condiciones de trabajo, tradicionalmente, se han considerado desde las estructuras de la legislación laboral en el cubrimiento de estos cuatro componentes: a) La seguridad social integral (salud, pensión y riesgos profesionales), contemplada en la Ley 100 de 1993, b) las prestaciones sociales y el derecho a las vacaciones, c) los entornos o ambientes laborales seguros, y d) el desarrollo personal y social de los trabajadores. Como se aprecia, las CT son un concepto complejo y dinámico que involucra diversos aspectos del contexto laboral, organizacional, de la seguridad social y de su bienestar, además de los sistemas de contratación, que es un aspecto importante en la organización laboral.

Investigaciones en Colombia, sobre las condiciones de trabajo de las enfermeras, reportan disminución de la satisfacción, aumento de la carga laboral y agotamiento, además de bajos salarios, precarias condiciones laborales y de seguridad social, poco reconocimiento por estudios de posgrado y distorsión del perfil de la enfermera, lo que se traduce en disminución de la calidad del cuidado ${ }_{15-22}$

Las CT en las enfermeras son dinámicas y han sufrido transformaciones con los movimientos del mercado laboral y los cambios en el sistema de salud colombiano; en consecuencia, se observan instituciones que ya tienen toda la planta de personal con las nuevas formas de contratación laboral.

Los estudios revisados entre el 2000 y el 2012 sobre este tema en Colombia, se realizaron en un periodo de transición en el que algunas enfermeras eran desvinculadas de las instituciones para iniciar una modalidad de contrato flexible. Ante este panorama en las formas de contratación, se realizó este estudio con el objetivo de describir las actuales condiciones de trabajo y de organización de los profesionales de enfermería de la ciudad. 


\section{Materiales y métodos}

Estudio descriptivo de corte transversal, con asociación de variables socio-demográficas de condiciones de trabajo y de organización de las enfermeras en las instituciones de salud de $2^{\circ}, 3^{\circ}$ y $4^{\circ}$ nivel de atención de una ciudad colombiana, realizado entre los años 2013 y 2014.

La población estuvo constituida por 2009 enfermeras (os) de la ciudad de Medellín y el Área Metropolitana con formación profesional, quienes a enero de 2013 estaban vinculadas a 31 Instituciones Prestadoras de Servicios de Salud (IPS).

Para la muestra se estableció el diseño muestral por conglomerados, en la primera parte se realizó un muestreo aleatorio simple, en la segunda parte se realizó una serie de conglomerados en función de la investigación para dar a todos la posibilidad de ser seleccionados.

La muestra estuvo representada por 552 enfermeras que laboran en las 31 instituciones de salud existentes, con un nivel de confianza del 95\% y un error del $4 \%$. El grupo investigador asumió una prevalencia del $50 \%$ dado que esto maximiza el tamaño muestral.

Los criterios de inclusión fueron: enfermeras con formación universitaria, mayores de edad, que tuvieran no menos de 3 meses de estar laborando con cualquier forma de contratación, y que voluntariamente desearan participar en el estudio. Fueron excluidas del estudio las enfermeras que gozaban de su pensión por vejez.

La información se recogió a través de la encuesta de datos socio-demográficos y del cuestionario de Condiciones de Trabajo $(q C T)$, previamente validado en Latinoamérica, y con la autorización de los autores. Este instrumento se compone de las variables de condiciones de trabajo que se configuran en torno a una triple relación de la organización con el método, con el entorno y con la persona; de igual forma, cada una de las tres dimensiones se divide en dos escalas para un total de seis escalas: ${ }_{24}$

1. Escala de organización y método: Regulación (6 ítems) que evalúa aspectos de las condiciones de trabajo como la calidad del contrato, la jornada, salarios, ritmos, descansos y la carga de trabajo, entre otros.

2. Escala de organización y método: Desarrollo (8 ítems) evalúa aspectos de las relaciones del trabajador con la dirección como: autonomía, justicia, oportunidades, participación, apoyo y evaluación del desempeño, entre otros.

3. Escala de organización y entorno material (4 ítems) evalúa aspectos de entorno físico del trabajo como: instalaciones, equipamiento, recursos, limpieza, seguridad y prevención de factores de riesgos.

4. Escala de organización y entorno social (4 ítems): evalúa las relaciones del trabajador (comunicación, respeto, reconocimiento) con su compañeros de trabajo, colegas y los usuarios.

Para las cuatro escalas anteriores las posibles categorías de respuesta de cada uno de los ítems van de un valor 0 a 10 donde 0 es pésimo y 10 es óptimo.

5. Escala de organización y persona: Ajuste (15 ítems) evalúa aspectos relacionados con la satisfacción en el trabajo como: la motivación, interés, valoración, respuesta a las expectativas y necesidades, crecimiento profesional y personal y la identidad entre otros.

6. Adaptación personal-organización (7 ítems): evalúa la actitud del trabajador de adaptación, aceptación, pertenencia e identidad ante las demandas de la organización, políticas, valores, protocolos, tiempos y ritmos de trabajo.

Para las dos últimas escalas las posibles categorías de respuesta de cada uno de los ítems van de un valor de 1 a 7 , donde 1 es total desacuerdo y 7 es total acuerdo.

En cuanto a las consideraciones éticas, se garantizaron los principios de anonimato, de confidencialidad, de respeto y de beneficencia a las participantes y a las instituciones. También se tuvo en cuenta el consentimiento informado de los participantes antes de realizar las encuestas. ${ }_{25}$ El estudio fue aprobado por el Comité de Ética de la Facultad de Enfermería de la Universidad de Antioquia.

Para el análisis estadístico de los datos se empleó el programa SPSS VR 19 (SPSS Inc. Chicago, Illinois, USA). Para el análisis descriptivo de los datos socio- 
demográficos se construyeron, en primera instancia, las tablas de frecuencias univariadas de cada una de las variables incluidas en el estudio. Adicionalmente, se diseñaron las tablas de contingencia bivariadas de mayor interés para establecer la existencia o no de asociación estadística, como la chi cuadrado y para las condiciones de trabajo y de organización por escalas se estableció, en cada una de las seis escalas, el comportamiento promedio de las enfermeras.

\section{Resultados}

El sexo femenino, con $87,2 \%$, predominó entre los participantes del estudio, 55,1\% tenía entre 25 y 34 años de edad, $52 \%$ eran solteras, $42,6 \%$ cabeza de familia, $49,8 \%$ pertenecían al estrato socio-económico tres, $78,3 \%$ eran egresadas de instituciones educativas públicas, 18,3\% con especialización y 1,4\% con maestría. El 63,3\% laboraba en instituciones privadas y $51.3 \%$ en instituciones de III y IV nivel de complejidad en salud. El 51,3 \% tenía contrato a término indefinido y $28,6 \%$ contrato flexible o con intermediación laboral (que incluye contrato sindical, cooperativa (CTA), prestación de servicios y empresa de servicio temporal).
El 33,5\% de las enfermeras devengaba salarios al mes entre $\$ 2.500 .000$ y $\$ 2.999 .000$ y $29 \%$ entre $\$ 2.000 .000$ y $2.499 .000 ; 83 \%$ contaba con seguridad social y todas las prestaciones sociales; $38,4 \%$ de los participantes tenían de 1 a 3 años de antigüedad en las instituciones y 19,6\% de 3 a 11 meses, 42,5\% laboraba en el servicio de hospitalización, 62\% realizaba funciones combinadas: administrativas y asistenciales, y $28,6 \%$, primordialmente asistenciales; $70 \%$ cumplía su jornada laboral en horario combinado diurno y nocturno, y solo en horario diurno 28,6\%. El 75,7\% de las enfermeras reportan horas no reconocidas en su jornada laboral y disponen de un departamento de enfermería solo el $56 \%_{38}$

\begin{abstract}
Asociaciones entre los tipos de contrato y las variables socio-demográficas. Al comparar las variables socio-demográficas con todos los tipos de contratación predominante: a término indefinido y contratación flexible, se encontró significancia estadística en las variables de tipo de institución, antigüedad laboral, cubrimiento de prestaciones sociales y salario mensual básico que afectaban las condiciones laborales de las enfermeras del estudio (Tabla 1).
\end{abstract}

Tabla 1. Asociación entre los tipos de contrato y variables socio-demográficas

\begin{tabular}{|c|c|c|c|c|c|c|c|}
\hline \multicolumn{8}{|c|}{ Tipo de contrato } \\
\hline \multicolumn{2}{|c|}{ Variable } & Fijo & \multirow{2}{*}{$\begin{array}{r}\text { Indefinido } \\
32\end{array}$} & \multirow{2}{*}{$\begin{array}{r}\text { Flexible } \\
80\end{array}$} & \multirow{2}{*}{$\begin{array}{r}\text { Total } \\
136\end{array}$} & \multirow[t]{2}{*}{$X^{2}$} & \multirow[t]{2}{*}{ P Valor } \\
\hline TIP & Pública & 24 & & & & & \\
\hline Tipo & Privada & 87 & 249 & 15 & 351 & & \\
\hline institución & Mixta & 0 & 2 & 63 & 65 & 315,62 & $<0,0001$ \\
\hline \multirow{6}{*}{$\begin{array}{l}\text { Antigüedad } \\
\text { laboral }\end{array}$} & $3-11$ meses & 34 & 16 & 58 & 108 & & \\
\hline & 1 - 3 años & 53 & 96 & 63 & 212 & & \\
\hline & $4-6$ años & 12 & 53 & 19 & 84 & & \\
\hline & 7 - 9 años & 6 & 24 & 10 & 40 & 124,1 & $<0,000$ \\
\hline & 10 - 12 años & 3 & 21 & 2 & 26 & & \\
\hline & 13 o más años & 3 & 72 & 6 & 81 & & \\
\hline \multirow{5}{*}{$\begin{array}{c}\text { Cubrimiento } \\
\text { prestaciones } \\
\text { sociales }\end{array}$} & Afil_S.Soc_Int* & 11 & 26 & 50 & 87 & & \\
\hline & Afil_S.Soc_Int+P** & 11 & 16 & 28 & 55 & & \\
\hline & Todas & 88 & 232 & 63 & 383 & & \\
\hline & Ninguna & 0 & 0 & 9 & 9 & 107,1 & $<0,0001$ \\
\hline & No sabe & 0 & 2 & 5 & 7 & & \\
\hline
\end{tabular}




\begin{tabular}{|c|c|c|c|c|c|c|c|}
\hline & \multicolumn{7}{|c|}{ Tipo de contrato } \\
\hline & Variable & Fijo & Indefinido & Flexible & Total & $X^{2}$ & P Valor \\
\hline \multirow{6}{*}{$\begin{array}{c}\text { Salario } \\
\text { mensual básico } \\
\text { (miles) }\end{array}$} & Menos 999 & 0 & 3 & 0 & 3 & & \\
\hline & 1000-1499 & 0 & 14 & 9 & 23 & & \\
\hline & 1500-1999 & 4 & 4 & 37 & 45 & 174,2 & $<0,0001$ \\
\hline & $2000-2499$ & 50 & 40 & 70 & 160 & & \\
\hline & 2500-2999 & 41 & 117 & 27 & 185 & & \\
\hline & 3000 -o-mas & 16 & 103 & 13 & 132 & & \\
\hline
\end{tabular}

*Afiliación a la seguridad social integral. ** Afiliación a la seguridad social integral más prestaciones sociales.

En el contrato flexible predominaban las enfermeras con menor antigüedad laboral de 3 - 11 meses (37\%), laboran en instituciones de carácter público $(50,6 \%)$, con un rango de salario de $\$ 2.000 .000-2.499 .000$ $(44,3 \%)$ y le cubren la seguridad social integral y todas las prestaciones sociales $(39,8 \%)$.

En cuanto al contrato a término indefinido predominan las enfermeras que laboran en empresas de carácter privado (88\%), con antigüedad laboral mayor de 10 años (32,8\%), devengan salarios entre $\$ 2.500 .000$ - $2.999 .000(41,3 \%)$ y con cubrimiento total de la seguridad social integral y prestaciones sociales el $82 \%$ ( Tabla 1 ).
Escalas de condiciones de trabajo y organización. El cuestionario de qCTalcanzó un Alpha de Crombach de $0,97 \%$, para todas las escalas y dimensiones, lo que demostró que el cuestionario es de alta confiabilidad y valida su utilización en nuestro medio. Las enfermeras del estudio, en general, y según la media y desviación estándar (Tabla 2), manifestaron una percepción positiva de las condiciones de trabajo en todas las escalas: regulación, desarrollo, entorno material y entorno social, ajuste y adaptación.

Tabla 2. Promedios por escala y desviación estándar

\begin{tabular}{llrrrr}
\hline \multicolumn{1}{c}{ Escala } & \multicolumn{3}{c}{ Promedios escalas } \\
\hline & & Media & DE & Mínimo & Máximo \\
1. & Organización y método: regulación & 7,01 & 1,76 & 1,00 & 10,00 \\
2. & Organización y método: desarrollo & 6,62 & 2,05 & 0,00 & 10,00 \\
3. & Organización y entorno material & 7,90 & 1,82 & 1,25 & 10,00 \\
4. & Organización y entorno social & 7,75 & 1,57 & 1,00 & 10,00 \\
5. & Ajuste organización- persona & 5,59 & 1,13 & 1,00 & 7,00 \\
6. & Adaptación persona- organización & 6,27 & 0,77 & 1,00 & 7,00 \\
\hline
\end{tabular}

Las escalas que obtuvieron mayor calificación en la media (Tabla 2), fueron entorno material (M 7,90, DE: 1,82 ) y la de adaptación (M 6,27, DE 0,77) y las de menor calificación fueron las escala de desarrollo (M 6,62 , DE 2,05) y la de ajuste (M 5,59, DE 1,13).

Al revisar todas las variables (Tabla 3) contempladas en las escalas 1 a 4 , las que obtuvieron la mayor calificación promedio, fueron: disponer de servicios auxiliares en la institución como son limpieza, seguridad, entre otros (M 8,04; entorno material) y reconocimiento del propio trabajo por personas usuarias (M 8,04; entorno social). Las variables de menos calificación promedio fueron: participación en las decisiones organizacionales (M 5,79; desarrollo) y carga de trabajo (M 6,33; regulación). Para las 
escalas 5 y 6, las variables de la escala de adaptación obtuvieron mayor calificación promedio comparadas con la escala de ajuste, se destaca: interiorizo fácilmente las normas establecidas en mi servicio ( $M$ 6,41; adaptación) y la de menor calificación promedio: me da sensación de libertad (M 5,15; ajuste).

Tabla 3. Promedios de variables en las escalas del cuestionario de qCT

\begin{tabular}{|c|c|c|c|}
\hline Escalas & Variable & Media & $\boldsymbol{\sigma}$ \\
\hline \multirow{8}{*}{ Regulación } & Tiempo de trabajo (horarios, ritmos, descansos, etc.) & 7,05 & 2,16 \\
\hline & Organización general del trabajo & 7,40 & 1,83 \\
\hline & Retribución económica & 7,05 & 2,34 \\
\hline & Carga de trabajo & 6,33 & 2,56 \\
\hline & Calidad del contrato laboral & 7,59 & 2,58 \\
\hline & Conciliación trabajo - vida privada y familiar & 6,64 & 2,53 \\
\hline & Autonomía en la toma de decisiones profesionales & 6,96 & 2,19 \\
\hline & Justicia en la contratación, remuneración y promoción & 6,91 & 2,45 \\
\hline \multirow{7}{*}{ Desarrollo } & Oportunidades para la formación continua & 6,61 & 2,72 \\
\hline & Vías de promoción laboral & 6,12 & 2,61 \\
\hline & Participación en las decisiones organizacionales & 5,79 & 2,68 \\
\hline & Relaciones con la dirección & 6,83 & 2,58 \\
\hline & Evaluación del rendimiento profesional por la institución & 6,99 & 2,28 \\
\hline & Apoyo recibido del personal directivo & 6,74 & 2,59 \\
\hline & Entorno físico, instalaciones y equipamientos & 7,83 & 2,09 \\
\hline \multirow{4}{*}{ Entorno Material } & Recursos materiales y técnicos & 7,96 & 1,98 \\
\hline & Prevención de riesgos laborales & 7,79 & 2,12 \\
\hline & Servicios auxiliares (limpieza, seguridad, etc.) & 8,04 & 1,90 \\
\hline & Compañerismo & 7,54 & 1,95 \\
\hline Entorno & Respeto en el grupo de trabajo & 7,93 & 1,78 \\
\hline \multirow[t]{9}{*}{ Social } & Reconocimiento del propio trabajo por colegas & 7,39 & 2,06 \\
\hline & Reconocimiento del propio trabajo por personas usuarias & 8,12 & 1,69 \\
\hline & Satisface mis intereses & 5,54 & 1,27 \\
\hline & Me exige según mis capacidades & 5,94 & 1,23 \\
\hline & Responde a mis necesidades & 5,61 & 1,31 \\
\hline & Encaja con mis expectativas & 5,53 & 1,41 \\
\hline & Se ajusta a mis aspiraciones & 5,40 & 1,43 \\
\hline & Concuerda con mis valores & 5,94 & 1,27 \\
\hline & Facilita que mis méritos sean valorados con justicia & 5,22 & 1,48 \\
\hline \multirow[t]{5}{*}{ Ajuste } & Estimula mi compromiso laboral & 5,33 & 1,52 \\
\hline & Me permite trabajar a gusto & 5,55 & 1,39 \\
\hline & Me motiva a trabajar & 5,46 & 1,43 \\
\hline & Me da sensación de libertad & 5,15 & 1,55 \\
\hline & Me hace crecer personalmente & 5,70 & 1,38 \\
\hline
\end{tabular}




\begin{tabular}{|c|c|c|c|}
\hline Escalas & Variable & Media & $\boldsymbol{\sigma}$ \\
\hline \multirow{6}{*}{ Ajuste } & Me permite desarrollar mis competencias profesionales & 5,79 & 1,28 \\
\hline & Me proporciona identidad & 5,72 & 1,31 \\
\hline & Me hace sentir útil & 6,04 & 1,19 \\
\hline & Me adapto a la política del centro & 6,18 & 1,03 \\
\hline & Asumo los valores de la dirección & 6,38 & 0,88 \\
\hline & Acepto que me digan cómo debo hacer mi trabajo & 6,26 & 0,90 \\
\hline \multirow{4}{*}{ Adaptación } & Interiorizo fácilmente las normas establecidas en mi servicio & 6,41 & 0,85 \\
\hline & Me identifico con el espíritu de los cambios propuestos & 6,21 & 1,03 \\
\hline & Aplico el protocolo ante cualquier dilema de conciencia & 6,19 & 1,04 \\
\hline & Me ajusto a los tiempos y ritmos de trabajo fijados & 6,29 & 1,03 \\
\hline
\end{tabular}

\section{Discusión}

Sexo, edad y cabeza de familia. Los datos que reporta el estudio sobre sexo, edad, cabeza de familia, estrato socio-económico y tipo de institución formadora de los participantes son similares a los reportados en el estudio de ACOFAEN realizado en el 2009 con población general de enfermeras egresadas de las principales universidades de Colombia. ${ }_{26}$

Entre los profesionales de enfermería predomina el sexo femenino $(87,2 \%)$ en razón, principalmente, de su tradición histórica, no obstante es inferior a lo encontrado en otros estudios y al promedio del registro de ANEC con un 92\%, se observa un incremento creciente y gradual de la población masculina de menor edad y con antigüedad laboral menor de 3 años. $_{15,18,21,27}$

El promedio de edad que predominó entre las enfermeras osciló entre 25 y 34 años (55,1\%), lo que muestra que las enfermeras son relativamente jóvenes, similar a los hallazgos de Restrepo y Ortiz; ${ }_{28}$ las de menor edad (20-24 años) están en el servicio o área de hospitalización, y las mayores (de más de 30 años) están distribuidas en todas las áreas, esto, en razón de que el servicio de hospitalización es el área que absorbe a la mayoría del personal de enfermería, y allí se ubican las recién egresadas, las otras áreas, más especializadas, requieren de profesionales con más antigüedad y experiencia para su desempeño.

De otro lado, $42,7 \%$ de las participantes se autodenominan cabeza de familia o como únicas proveedoras, dato que resulta superior al del promedio nacional de la ANEC que reporta un $27 \%{ }_{27}$
Formación en posgrado. El porcentaje de enfermeras con especialización fue de 18,3\%, inferior al del estudio de ACOFAEN, ${ }_{26}$ el cual reporta un $24 \%$ y al de Herrera y Manrique ${ }_{18}$ un $33 \%$.

Ahora bien, el hecho de que las enfermeras del estudio tuvieran una especialización, no hizo que cambiara su función o su desempeño en relación con las que no la tienen. Sin embargo, el bajo porcentaje de enfermeras con posgrados es preocupante (55\%), toda vez que se desempeñan en instituciones que, por su alta complejidad requieren de personal calificado; las que lo tienen, asumen un rol más que todo, administrativoasistencial; cabe anotar, además, que a las enfermeras no se les reconoce con salario esta nueva titulación, lo que pudiera desmotivarlas por falta de apoyo laboral e institucional para cualificarse. ${ }_{26}$

Antigüedad laboral. La antigüedad laboral de 57,1\% (en la última empresa contratante) en las enfermeras es menor a 3 años, lo que indica una alta rotación de empleos entre las de mayor edad, y en las más jóvenes, su primer empleo, que se cambia con frecuencia, en busca de mejores condiciones de trabajo. ${ }_{10}$

Este estudio muestra que a medida que se obtiene antigüedad laboral (mayor de 13 años) se desempeñan en el área administrativa y en instituciones privadas, por ser las que ofrecen mayor estabilidad y mejores salarios (superiores a \$2.500.000), además de otros incentivos que repercuten en la calidad de la atención y en la continuidad de los procesos técnicos y administrativos. ${ }_{13}$ 
Tipos de contratación. En Colombia la contratación flexible está en incremento y desplazando los contratos tradicionales a término fijo y a término indefinido. En este estudio la contratación flexible o con intermediación laboral $(28,6 \%)$, es inferior al promedio nacional de la $\mathrm{ANEC}_{27}$ que reporta $50 \%$; siendo las empresas públicas y mixtas las que más la aplican. ${ }_{10-13}$ En la contratación flexible la relación entre la administración, las enfermeras y los objetivos misionales no es clara y por esta razón reportan mayores dificultades. En Latinoamérica también se observa el incremento de este tipo de contratación con deterioro de las condiciones de trabajo y de organización en las instituciones. ${ }_{29,30}$

Salarios. Los mayores ingresos salariales (superiores a 3 millones al mes) están relacionados con los contratos a término indefinido, el desempeño en áreas administrativas y servicios complementarios de alta tecnología; el menor ingreso $44,3 \%$ con un salario entre $\$ 2.000 .000-2.499 .000$, se da en el sistema de contrato flexible, (Tabla 1). Pero, en general, los rangos de salario que muestra el estudio son superiores al promedio nacional; la $\mathrm{ANEC}_{27}$ reporta que $51 \%$ de las enfermeras devenga salarios inferiores a $\$ 1.500 .000 \mathrm{al}$ mes.

Independiente del tipo de contrato laboral, 75,7\% de las enfermeras que participaron en este estudio reportan horas no reconocidas salarialmente, el $32 \%$ entre $1-4$ horas por semana y el $27 \%$ entre 5-8. Similar a los hallazgos de un estudio multicéntrico en Latinoamérica que dan cuenta de que las enfermeras laboran más de 48 horas a la semana, y son las que más horas de trabajo realizan fuera de su jornada laboral $_{31}$

Funciones y organización. Cuando las participantes hacen funciones administrativas y al mismo tiempo asistenciales $(61,6 \%)$, le restan tiempo al cuidado directo (asistencial) del paciente y la familia, que solo alcanza 28,6\%, como lo señala, también, el estudio de García et $\mathrm{al}_{15}$ situación que afecta negativamente la gestión del cuidado, la satisfacción laboral y la pertenencia a la institución. ${ }_{22,20}$

El $56,1 \%$ de las enfermeras cuenta con un departamento de enfermería, representado por 13 IPS privadas y una IPS pública. Las IPS privadas de reciente constitución, al igual que las públicas y mixtas no lo tienen, en su lugar, disponen de un coordinador médico y un coordinador de enfermería o líder de procesos, asunto que debe inquietar a estos profesionales ya que en la administración y organización del personal de enfermería se hace necesaria la jefatura y el departamento de enfermería con autonomía administrativa para la gestión del recurso humano y del cuidado ${ }_{32}$

Este estudio demuestra que las enfermeras de la ciudad de Medellín y su área Metropolitana que trabajan con contrato a término indefinido y en instituciones de carácter privado tienen mejores condiciones laborales (cubrimiento de seguridad social y prestaciones sociales, antigüedad laboral y salarios), que las que trabajan con contrato flexible (Tabla 1), si se comparan con el promedio nacional, esto hace que enfermeras de otras regiones del país como de la Costa Atlántica, Chocó y el Eje Cafetero migren a esta ciudad en busca de empleo, como se evidenció en los estudios de ACOFAEN y ANEC. ${ }_{26,27}$

Se constata también en este trabajo que las IPS públicas y mixtas son las que más vinculan enfermeras jóvenes y con poca experiencia con la modalidad de contrato flexible (contrato sindical, cooperativa y prestación de servicios, entre otros), situación que sería favorable si estas enfermeras ganaran en antigüedad y cualificación profesional; pero, por el contrario, lo que se observa es una alta movilidad de estas profesionales en busca de empleo, desmotivadas por los bajos salarios y la falta de cubrimiento de prestaciones sociales (recargo de horas en nocturno y festivos, primas y vacaciones, entre otros), con consecuencias negativas no solo para ellas (frustración, falta de estabilidad y satisfacción laboral), sino también para las instituciones por la falta de continuidad en los procesos y sus implicaciones en la calidad de la atención.

Otra situación preocupante es el desconocimiento de los derechos laborales por parte de las participantes como el tipo de contrato, seguridad social, prestaciones sociales y datos específicos de la institución en la que laboran, entre otras razones, por los cambios de la razón social de algunas instituciones contratantes, lo que contradice los planteamientos de la filosofía y políticas de los sindicatos que anuncian asociaciones gremiales de profesionales para promover y defender el trabajo digno. 
Finalmente, llama la atención que una IPS de carácter público y mixto, que debería dar ejemplo de contratación garante de los derechos laborales, es justamente este tipo de instituciones en donde se ponen en entredicho las condiciones de trabajo dignas. En esta ciudad predomina la contratación de IPS privadas en la modalidad de contrato indefinido, no obstante, ya empieza a vislumbrarse la aparición de estas nuevas IPS y con ellas la modalidad de contratación flexible.

En Colombia son pocos los estudios que analizan las condiciones de trabajo y la organización desde instrumentos validados, se encontró estudios sobre satisfacción laboral, calidad de vida laboral, desgaste laboral y riesgo psicosocial que contemplan algunos componentes relacionados en las escalas del estudio. En Norteamérica y Europa se encuentran más estudios sobre la organización de las enfermeras.

Las enfermeras del estudio califican el entorno material y social con los mayores promedios, lo que indica que las IPS donde laboran, lo garantizan. Lo que más valoran es disponer de servicios de apoyo en el entorno laboral para su comodidad y seguridad en su desempeño. ${ }_{33}$ Así mismo, el que sean reconocidas por los usuarios y por el equipo de salud, es muy valorado y esto reafirma su satisfacción laboral e identidad profesional en la institución. ${ }_{34}$

Las enfermeras identifican poca participación en las decisiones organizacionales, no son tenidas en cuenta, lo que afecta su reconocimiento y autonomía en la institución, aunado a una alta carga de trabajo, como se evidencia en otros estudios ${ }_{35,36}$ Esta situación es contraria a lo encontrado en Norteamérica, donde se les da participación en los procesos administrativos de la empresa, se promueve estrategias para retener a las enfermeras con estímulos de competencias profesionales, capacitaciones y cumplimiento de indicadores de productividad y seguridad para los

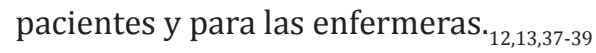

El estudio reporta menor calificación en la escala de ajuste, al compararla con la de adaptación. En la escala de ajuste, las enfermeras inicialmente hacen una reflexión personal y una calibración entre lo que la institución les ofrece y los intereses personales y expectativas laborales, esto es determinante para el logro de una adaptación positiva en la institución y la adherencia a las políticas, normas y protocolos. Cuando las enfermeras no se adaptan, están insatisfechas y hacen todo lo posible por cambiar de empleo, como se observó en el estudio, el 58\% de las enfermeras tienen una antigüedad menos a 3 años en su último empleo, las más afectadas en la movilidad son las más jóvenes y las que laboran en contratos de modalidad flexible.

El recurso humano de enfermería demanda unas condiciones de trabajo y de organización digna, saludable y satisfactoria, para garantizar un desempeño con criterios de calidad, por esto es necesario continuar con estos estudios para tener pruebas e indicadores del comportamiento de las condiciones de trabajo y de organización de las enfermeras en las instituciones.

\section{Conclusiones}

Las enfermeras del estudio, según la media y desviación estándar manifestaron una percepción positiva de las condiciones de trabajo en todas las escalas, no obstante al analizar las escalas con algunas variables de interés se observan diferencias significativas para su interpretación.

Las variables de tipo de contrato, tipo de empresa, antigüedad laboral, salarios y el cubrimiento de la seguridad social y prestaciones sociales son determinantes para la calificación de las escalas de $q C T$.

Las mejores condiciones de trabajo y de organización: mayor antigüedad laboral, mayor salario, cubrimiento total de la seguridad social y prestaciones sociales, se dan en las enfermeras que laboran en contrato a término indefinido y en empresas de carácter privado, por el contrario las enfermeras que laboran en empresas de carácter público o mixto y con el contrato flexible reportan condiciones de trabajo menos favorables: menor antigüedad laboral, menor salario y cubrimiento parcial de la seguridad social y prestaciones sociales.

El grupo de enfermeras más jóvenes (20 y 29 años) y de reciente incursión a su vida laboral reportan mayor vulnerabilidad para las condiciones de trabajo, en él predomina la contratación flexible. 


\section{Referencias}

1. Beck U. The brave new world of work. Cambridge: Polity Press; 2000. Sociology [Internet]. 2001 Nov [citado 2014 Nov 5];35(04):991-1008. Disponible en: http://journals.cambridge.org/abstract_ S0038038501219919

2. Aguilar MA, Rei N (Coords.). Tratado de psicología social. Perspectivas socioculturales. Barcelona: Anthropos; 2007:210-38.

3. Davis K, Newstrom JW. El comportamiento humano en el trabajo. Comportamiento organizacional. México: Mc GrawHill, Interamericana de México; 1991:206-7.

4. Cortes R, Ramírez A, Restrepo M, Vargas B. Declaración del Consejo Técnico Nacional de Enfermería sobre el deterioro de las condiciones laborales del profesional de enfermería en Colombia. Actual Enferm [Internet]. 2010 [citado 2014 Nov 5];13(3):28-35. Disponible en: http://encolombia.com/medicina/revistas-medicas / enfermeria/vole-134/declaraciondelconsejotecnico/

5. Arenas-Gallego E, Piedrahíta-Vargas C, Plata-López JM. Marco jurídico de las cooperativas de trabajo asociado. Opin Jurid [Internet]. 2007 [citado 2014 Nov 5]; 6(11):33-45. Disponible en: http://www.scielo. org.co/scielo.php?script=sci_arttext $\&$ pid $=$ S1692$25302007000100002 \& \operatorname{lng}=e n \& n r m=i s o \& \operatorname{lng}=e s$

6. López-López P. Modernización, flexibilización, sumisión: las condiciones laborales en la sociedad de la información. El viejo topo [Internet]. $2000 \mathrm{Sep}$ [citado 2014 Nov 5];(143):37-43. Disponible en: http://eprints.ucm.es/9784/

7. Vidal P. Una aproximación a las condiciones laborales de trabajo social: un insumo para el debate en la actualidad del ejercicio profesional. Rev Perspectivas [Internet]. 2008 [citado 2014 Nov 5];19:129-55. Disponible en: http://ww3.ucsh.cl/resources/ descargas/landing/revistas/Perspectivas_19.pdf

8. Tomasina F, Bozzo E, Chaves E, Pucci F. Impacto de las condiciones laborales en la salud de trabajadores de un centro quirúrgico. Rev Cub Salud Pública [Internet]. 2008 [citado 2014 Nov 5];34(2) Disponible en: http:// www.redalyc.org/resumen.oa?id=21419850008

9. Mauro MYC, Paz AF da, Mauro CCC, Pinheiro MA de S, Silva VG. Condições de trabalho da enfermagem nas enfermarias de um hospital universitário. Esc Anna Nery [Internet]. 2010 Jun [citado 2014 Nov 5];14(2):244-52. Disponible en: http://www. scielo.br/scielo.php?script=sci_arttext\&pid=S1414$81452010000200006 \& \operatorname{lng}=e n \& n r m=i s o \& \operatorname{lng}=p t$
10. Malvárez SM, Castrillón MC. Panorama de la fuerza de trabajo en enfermería en América Latina. Primera parte. Rev Enferm IMSS [Internet]. 2006 [citado 2014 Nov 5]; 14 (2):101-16. Disponible en: http://www. medigraphic.com/pdfs/enfermeriaimss/eim-2006/ eim062g.pdf

11. Baraldi S, Car MR. Labor flexibilization and deregulation for nursing workers in Brazil: the PROFAE case. Rev Lat Am Enfermagem [Internet]. $2008 \mathrm{Abr}$ [citado 2014 Nov 5];16(2):205-11. Disponible en: http://www. ncbi.nlm.nih.gov/pubmed/18528586

12. Mark BA, Lindley L, Jones CB. Nurse working conditions and nursing unit costs. Policy Polit Nurs Pract [Internet]. 2009 May [citado 2014 Nov 5];10(2):1208. Disponible en: http://www.pubmedcentral.nih.gov/ articlerender.fcgi?artid=2923486\&tool=pmcentrez\&r endertype $=$ abstract

13. Stone PW, Mooney-Kane C, Larson EL, Horan T, Glance LG, Zwanziger J, et al. Nurse working conditions and patient safety outcomes. Med Care [Internet]. 2007 Jun [citado 2014 Nov 6];45(6):571-8. Disponible en: http://www.ncbi.nlm.nih.gov/pubmed/17515785

14. Consejo Internacional de Enfermería. Entornos positivos para la práctica de los profesionales de atención de salud. [Internet]. Geneva-Switzerland; 2008; Available from: http://www.icn.ch/images/ stories/documents/pillars/sew/ICHRN/Facts_ Sheets/Entornos_positivos_para_la_practica_de_los_ profesionales_de_atencion_de_salud.pdf

15. Garcia-Ospina C, López-Ríos L, Osorio-Gómez L, RealpeDelgado C. Desempeño profesional de los egresados del programa de enfermería de la Universidad de Caldas y su relación con la Ley de Seguridad Social en Salud y con el perfil de formación (Manizales 1995 y 2004). Hacia Promoc Salud [Internet]. 2007 [citado 2014 Nov 5];12:91-108. Disponible en: http://www. scielo.org.co/pdf/hpsal/v12n1/v12n1a07.pdf

16. Cogollo-Milanés Z, Batista E, Cantillo C, Jaramillo A, Rodelo D, Meriño G. Desgaste profesional y factores asociados en personal de enfermería de servicios de urgencias de Cartagena, Colombia. Aquichan [Internet]. 2010 [citado 2014 Nov 5];10(1):43-51. Disponible en: http://www.redalyc.org/resumen. oa?id=74116244005

17. Florez-Acosta JH, Atehortúa-Becerra SC, Arenas-Mejía AC. Las condiciones laborales de los profesionales de la salud a partir de la Ley 100 de 1993: evolución y un estudio de caso para Medellín. Rev Gerenc Polit Salud [Internet]. 2009 Jan-Jun [citado 2014 Nov 5];8(16):107-31. Disponible en: http://www.scielo. org.co/scielo.php?script=sci_arttext $\&$ pid=S1657$70272009000100007 \& \operatorname{lng}=\mathrm{en} \& \mathrm{nrm}=\mathrm{iso} \& \operatorname{tng}=\mathrm{es}$ 
18. Herrera-Amaya G, Manrique-Abril FG. Condiciones laborales y grado de satisfacción de profesionales de enfermería. Aquichán [Internet]. 2008 Oct [citado 2014 Nov 5];8(2):243-56. Disponible en: http://www.scielo.org.co/scielo.php?script=sci_ arttext\&pid=S1657-59972008000200011\&lng=es\&n $\mathrm{rm}=\mathrm{iso} \& \mathrm{t} \operatorname{lng}=\mathrm{es}$

19. Guerrero J, Pulido G. Trabajo, salud y régimen contractual en personal de enfermería: un enfoque psicosocial. Av Enferm [Internet]. 2010 [citado 2014 Nov 5];28(2):111-22. Disponible en: http://www. revistas.unal.edu.co/index.php/avenferm/article/ view/21386/22355

20. Moreno MN, Mesa L, Galindo S. Qualidade de vida das(os) profissionais da enfermagem e suas conseqüências na atenção. Av Enferm [Internet]. [citado 2014 Nov 5];26(2):57-68. Disponible en: http://bases.bireme.br/cgi-bin/wxislind.exe/iah/ online/?IsisScript=iah/iah.xis\&src=google\&base=LIL ACS\&lang=p\&nextAction=lnk\&exprSearch=528693\&i ndexSearch $=$ ID

21. Cogollo-Milanés Z, Gómez-Bustamante E. Condiciones laborales en Cartagena, Colombia. Av Enferm [Internet]. 2010 Jun [citado 2014 Nov 5];28(1):31-8. Disponible en: http://www.scielo.org.co/scielo.php?script=sci_ arttext\&pid=S0121-45002010000100004\&lng=en\&n $\mathrm{rm}=\mathrm{iso} \& \operatorname{tng}=\mathrm{es}$

22. Zapata-Herrera M, Alcaraz-López GM. Job satisfaction of nursing professionals in adult hospitalization wards. An ambivalent feeling. Invest Educ Enferm. [Internet]. 2012 [citado 2014 Nov 5];30(2):178-87. Disponible en: http:// http://aprendeenlinea.udea.edu.co/ revistas/index.php/iee/article/view/7879/11960

23. Colimon KM. Fundamentos de epidemiología. 3a ed. Medellín: CIB. Corporación para Investigaciones Biológicas; 2010. p. 176-177.

24. Blanch JM, Sahagún M, Cervantes G. Estructura factorial del cuestionario de condiciones de trabajo. Rev Psicol Trab Organ [Internet]. 2010 Dic [citado 2014 Nov 6];26(3):175-89. Disponible en: http://scielo. isciii.es /scielo.php?script=sci_arttext \&pid=S157659622010000300002\&lng=es\&nrm=iso\&tlng=es

25. Colombia. Ministerio de Salud. Resolución No. 8430 de 1993, por la cual se establecen las normas científicas, técnicas y administrativas para la investigación en salud. Bogotá: Ministerio de Salud; 1993 [Internet]. [citado 2014 Nov 6] Disponible en: http://www. unisabana.edu.co/fileadmin / Documentos / Investigacion/comite_de_etica/Res__8430_1993_-Salud.pdf
26. Asociación Colombiana de Facultades de Enfermería. Estudio multicéntrico: Ejercicio laboral y condiciones generales de trabajo de los profesionales de enfermería egresados entre 1995 y 2004 en Colombia en el contexto del Sistema General de Seguridad Social en Salud. Bogotá: ACOFAEN; 2009.

27. Prada-Moreno C. Asociación Nacional de Enfermeras de Colombia. Base de datos del RUN aporta indicadores socio laborales de profesionales de enfermería. Rev Anec [Internet]. 2014 Jul [citado 2014 Nov 6];78 :445. Disponible en: http://www.anec.org.co/revista78/ revista-78.pdf

28. Restrepo DA, Ortiz LC. Situación laboral de los profesionales en medicina y enfermería en Colombia: aproximaciones desde el Ingreso Base de Cotización al SGSSS. Ministerio de la Protección Social. Boletín No. 2 [Internet]. 2010 Dic [citado 2014 Nov 6];1-4. Disponible en: http://www.minsalud. gov.co/Documentos $\% 20 y \% 20$ Publicaciones / APROXIMAC I \% C 3\% 93 N \% 20 A L \% 20 I B C \% 20 PROFESIONALES\%20EN\%20MEDICINA\%20Y\%20 ENFERMER\%C3\%8DA.pdf

29. Novick M, Rosales C. Desafíos de la gestión de los recursos humanos en salud 2005-2015. Organizacion Panamericana de la Salud [Internet]. 2006 [citado 2014 Nov 6] Disponible en: http://cursos.campusvirtualsp. org/pluginfile.php/58642/mod_resource/content/1/ desafios-gestion-rh-esp.pdf

30. Uribe-Rodríguez AF, Garrido-Pinzón J, Rodríguez AM. Influencia del tipo de contratación en la calidad de vida laboral: manifestaciones del capitalismo organizacional. Revista Virtual Universidad Católica del Norte [Internet] 2011 May- Ago[citado 2014 Nov 5]; (33): 1-16. Disponible en: http://www.redalyc. org/pdf/1942/194218961007.pdf

31. Organización Panamericana de la Salud. Estudio comparativo de las condiciones de trabajo y salud de los trabajadores de la salud en: Argentina, Brasil, Costa Rica y Perú. Washington D.C: OPS; 2012 [Internet]. [citado 2014 Nov 6]. Disponible en: http://www. who.int/workforcealliance/knowledge/resources/ condicionestrabajo/es/

32. Peña-Riveros B, Vega-Vega Y. La Importancia de un espacio para enfermería en la organización institucional. Av Enferm [Internet]. 2003 Jul [citado 2014 Nov 5];21(2):5-16. Disponible en: http://www. enfermeria.unal.edu.co/revista/articulos/xxi2_1.pdf

33. Purdy N, Spence Laschinger HK,Finegan J, Kerr M, Olivera F. Effects of work environments on nurse and patient outcomes. J Nurs Manag [Internet]. 2010 nov 
[citado 2014 Nov 6]; 18(8): 901-13. Disponible en: http://www.ncbi.nlm.nih.gov/pubmed/21073564

34. Hinno S, Partanen P, Vehviläinen-Julkunen K. Hospital nurses' work environment, quality of care provided and career plans. Int Nurs Rev [Internet]. 2011 Jun [citado 2014 Nov 6]; 58(2): 255-62. Disponible en: http://www.ncbi.nlm.nih.gov/pubmed/21554301

35. Iliopoulou KK, While AE. Professional autonomy and job satisfaction: survey of critical care nurses in mainland Greece. J Adv Nurs [Internet]. 2010 Nov [citado 2014 Nov 6]; 66(11): 2520-31. Disponible en: http://www.ncbi.nlm.nih.gov/pubmed/20735503

36. Hu YC, Chen JC, Chiu HT, Shen HC, Chang WY. Nurses' perception of nursing workforce and its impact on the managerial outcomes in emergency departments. J Clin Nurs [Internet]. 2010 jun [citado 2014 Nov 6]; 19(11-12): 1645-53. Disponible en: http://www.ncbi. nlm.nih.gov/pubmed/20384667
37. Porter C. A nursing labor management partnership model. J Nurs Adm [Internet]. 2010 jun [citado 2014 Nov 6]; 40(6): 272-6. Disponible en: http://www.ncbi. nlm.nih.gov/pubmed/20502196

38. Albanese MP, Evans DA, Schantz CA, Bowen M, Disbot M, Moffa JS, Piesieski P, Polomano RC. Engaging clinical nurses in quality and performance improvement activities. Nurs Adm Q [Internet]. 2010 Jul-Sep [citado 2014 Nov 6]; 34(3): 226-45. Disponible en: http:// www.ncbi.nlm.nih.gov/pubmed/20562572

39. Mrayyan MT. Hospital organizational climates and nurses' intent to stay: differences between units and wards. Contemp Nurse; [Internet]. 2008 feb [citado 2014 Nov 6]; 27(2): 223-36. Disponible en: http:// www.ncbi.nlm.nih.gov/pubmed/18457522 